\title{
Rational expectations equilibria and the ex-post core of an economy with asymmetric information
}

\author{
Ezra Einy $^{\mathrm{a}}$, Diego Moreno $^{\mathrm{b}}, *$ Benyamin Shitovitz $^{\mathrm{c}}$ \\ a Department of Economics, Ben Gurion University of the Negev, Beer Sheva 84105, Israel \\ b Deparatmento de Economía, Universidad Carlos III de Madrid, 28903 Getafe, Spain \\ ${ }^{c}$ Department of Economics, University of Haifa, Haifa 31905, Israel
}

Received 5 March 1999; received in revised form 28 October 1999; accepted 22 May 2000

\begin{abstract}
We study the relationship between the set of rational expectations equilibrium allocations and the ex-post core of exchange economies with asymmetric information. (C) 2000 Elsevier Science
\end{abstract} S.A. All rights reserved.

Keywords: Rational expectations equilibria; Asymmetric information; Ex-post core

\section{Introduction}

The core of an exchange economy with complete information and its relationship to competitive allocations has been studied extensively in the literature (for a comprehensive survey, see Anderson, 1992). For economies with asymmetric information, several alternative notions of core had been proposed (e.g., Wilson, 1978; Kobayashi, 1980; Yannelis, 1991; Koutsougeras and Yannelis, 1993; Allen, 1997). In this paper, we study the relationship between the set of rational expectations equilibrium allocations and the ex-post core (i.e., the set of allocations which ex-post cannot be improved upon by any coalition) of an economy with asymmetric information.

We consider an exchange economy with asymmetric information in which the space of traders is a measure space, and the set of states of nature is finite. Our framework is closely related to that of Allen (e.g., Allen, 1981, 1986). In studies of rational expectations equilibria, it is common to appeal to an artificial family of complete information economies associated with the original economy; see, e.g., Grossman (1978, 1981), Radner (1979,

\footnotetext{
${ }^{*}$ Corresponding author. Tel.: +34-91-624-9653; fax: +34-91-624-9875.

E-mail addresses: einy@bgumail.bgu.ac.il (E. Einy),dmoreno@eco.uc3m.es (D. Moreno), binya@econ.haifa.ac.il (B. Shitovitz).
} 
1982), Allen $(1981,1982)$. We show that the ex-post core consists of all the selections from the core correspondence of the associated family of complete information economies. Consequently, every rational expectations equilibrium allocation corresponding to fully revealing equilibrium prices is in the ex-post core of the economy. This need not be the case when equilibrium prices are not fully revealing (see Example 4.2).

We also prove that when the utility function of every trader is measurable with respect to his information field (that is, when every trader knows his utility function), the set of rational expectations equilibrium allocations consists of all selections from the competitive equilibrium correspondence of the associated family of complete information economies. This result and the representation result for the ex-post core imply, using Aumann's Core Equivalence Theorem, see Aumann, 1964, that if the economy is atomless and the utility function of each trader is measurable with respect to his information field, then the set of rational expectations equilibrium allocations coincides with the ex-post core. We also show that when the utility function of each trader is measurable with respect to his information field, then an analog of Debreu-Scarf's Theorem, see Debreu and Scarf (1963), holds for rational expectations equilibrium and ex-post core allocations.

\section{The model}

We consider a pure exchange economy $\mathcal{E}$ with differential information. The space of the traders is a measure space $(T, \Sigma, \mu)$, where $T$ is a set (set of traders), $\Sigma$ is a $\sigma$-field of the subsets of $T$ (the set of coalitions), and $\mu$ is a measure on $\Sigma$. The commodity space is $\mathcal{R}_{+}^{l}$. The space of states of nature is a measurable space $(\Omega, \mathcal{F})$, where $\Omega$ is a finite set and $\mathcal{F}$ is a field of subsets of $\Omega$.

Traders do not necessarily know which state of nature $\omega \in \Omega$ actually occurred, although they know their own endowments, and may also have some additional information about the state of nature. We assume that the information of a trader $t \in T$ is described by a measurable partition $\Pi_{t}$ of $\Omega$. We denote by $\mathcal{F}_{t}$ the field generated by $\Pi_{t}$. If $\omega_{0}$ is the true state of nature, trader $t$ observes the member of $\Pi_{t}$ which contains $\omega_{0}$. Every trader $t \in T$ has a probability measure $q_{t}$ on $\mathcal{F}$, which represents his prior beliefs. For simplicity, it is assumed that if $A \in \mathcal{F}$ is a non-empty set, then $q_{t}(A)>0$ for all $t \in T$. The preferences of a trader $t \in T$ are represented by a state-dependent utility function, $u_{t}: \Omega \times \mathcal{R}_{+}^{l} \rightarrow \mathcal{R}$ such that for every $x \in \mathcal{R}_{+}^{l}$, the function $u_{t}(\cdot, x)$ is $\mathcal{F}$-measurable. It is also assumed that for every $(t, x) \in T \times \mathcal{R}_{+}^{l}$, the mapping $(t, x) \rightarrow u_{t}(\omega, x)$ is $\Sigma \times \mathcal{B}$-measurable, where $\omega$ is a fixed member of $\Omega$, and $\mathcal{B}$ is the $\sigma$-field of Borel subsets of $\mathcal{R}_{+}^{l}$. Traders' initial endowments are described by a function $\boldsymbol{e}: \Omega \times T \rightarrow \mathcal{R}_{+}^{l}$ such that for every $\omega \in \Omega, \boldsymbol{e}(\omega, \cdot)$ is $\mu$-integrable on $T ; \boldsymbol{e}(\omega, t)$ represents the initial endowment of trader $t \in T$ in the state of nature $\omega \in \Omega$.

Since $\Omega$ is finite, there is a finite subfamily $\left(\mathcal{F}_{i}\right)_{i=1}^{n}$ of $\left(\mathcal{F}_{t}\right)_{t \in T}$ such that for every $t \in T$, there is $1 \leq i \leq n$ with $\mathcal{F}_{t}=\mathcal{F}_{i}$. We assume that for all $1 \leq i \leq n$, the set $T_{i}=\left\{t \in T \mid \mathcal{F}_{t}=\mathcal{F}_{i}\right\}$ is measurable and $\mu\left(T_{i}\right)>0$. We also assume that $\mathcal{F}=\bigvee_{i=1}^{n} \mathcal{F}_{i}$, which means that $\mathcal{F}$ contains no superfluous events about which no trader has information, and therefore cannot affect anyone's consumption decisions.

We use the following notations. For two vectors $x=\left(x_{1}, \ldots, x_{l}\right)$ and $y=\left(x_{1}, \ldots, x_{l}\right)$ in $\mathcal{R}^{l}$, we write $x \geq y$ when $x_{k}>y_{k}$ for all $1 \leq k \leq l$, $x y$ when $x \geq y$ and $x \neq y$, and $x \gg y$ when $x_{k}>y_{k}$ 
for all $1 \leq k \leq l$. A function $u: \mathcal{R}_{+}^{l} \rightarrow \mathcal{R}$ is (strictly) increasing if for all $x, y \in \mathcal{R}_{+}^{l}$, $(x>y) x \gg y$ implies $u(x)>u(y)$; it is quasi concave if for all $x, y \in \mathcal{R}_{+}^{l}$ and $\alpha \in[0,1]$, $u(\alpha x+(1-\alpha) y) \geq \min \{u(x), u(y)\}$; and it is strictly quasi concave if for all $x, y \in \mathcal{R}_{+}^{l}$, $x \neq y$, and $\alpha \in(0,1), u(\alpha x+(1-\alpha) y)>\min \{u(x), u(y)\}$.

Throughout the paper, we will often refer to the following conditions.

(A.1). For every $\omega \in \Omega, \int_{T} \mathbf{e}(\omega, t) \mathrm{d} \mu \gg 0$.

(A.2). For every $t \in T$, the function $\boldsymbol{e}(\cdot, t)$ is $\mathcal{F}_{t}$-measurable.

(A.3). For every $x \in \mathcal{R}_{+}^{l}$ and $t \in T$, the function $u_{t}(\cdot, x)$ is $\mathcal{F}_{t}$-measurable.

(A.4). For every $t \in T$ and $\omega \in \Omega$, the function $u_{t}(\omega, \cdot)$ is continuous, strictly increasing, and quasi concave on $\mathcal{R}_{+}^{l}$.

(A.5). For every $t \in T$ and $\omega \in \Omega$, the function $u_{t}(\omega, \cdot)$ is continuous, increasing, and strictly quasi concave on $\mathcal{R}_{+}^{l}$.

Imposing (A.1) guarantees that at every state of nature every commodity is present in the market. (A.2) and (A.3) require that each agent knows, respectively, his (state dependent) initial endowments and utility function. Finally, (A.4) and (A.5) impose properties on the traders utility functions. (Note that (A.5) implies (A.4)).

In the rest of the paper, an economy $\mathcal{E}$ is an economy with asymmetric information as described above. For an economy $\mathcal{E}$ and a state of nature $\omega \in \Omega$, we denote by $\mathcal{E}(\omega)$ the complete information economy in which the commodity space is $\mathcal{R}_{+}^{l}$, the space of the traders is $(T, \Sigma, \mu)$, and for every trader $t \in T$, his initial endowment is $\boldsymbol{e}(\omega, t)$ and his utility function is $u_{t}(\omega, \cdot)$; also, we write $C(\mathcal{E}(\omega))$ for the core of $\mathcal{E}(\omega)$.

\section{The ex-post core}

In this section we define the ex-post core of an economy $\mathcal{E}$, and we show that under (A.1) and (A.4), it is a non-empty set. Furthermore, the ex-post core of an economy $\mathcal{E}$ consists of all the selections from the core correspondence of the associated family of complete information economies $\{\mathcal{E}(\omega)\}_{\omega \in \Omega}$.

Let $\mathcal{E}$ be an economy. An assignment is a function $x: \Omega \times T \rightarrow \mathcal{R}_{+}^{l}$ such that for every $\omega \in \Omega$, the function $\boldsymbol{x}(\omega, \cdot)$ is $\mu$-integrable on $T$, and for every $t \in T$, the function $\boldsymbol{x}(\cdot, t)$ is $\mathcal{F}$-measurable. An allocation is an assignment $\boldsymbol{x}$ such that $\int_{T} \boldsymbol{x}(\omega, t) \mathrm{d} \mu \leq \int_{T} \boldsymbol{e}(\omega, t) \mathrm{d} \mu$ for every $\omega \in \Omega$. Let $\boldsymbol{x}$ be an allocation, let $S \in \Sigma$ be a coalition, and let $\omega_{0} \in \Omega$; we say that an assignment $\boldsymbol{y}$ is an ex-post improvement of $S$ upon $\boldsymbol{x}$ at $\omega_{0}$ if

(3.1) $\mu(S)>0$,

(3.2) $\int_{S} \boldsymbol{y}\left(\omega_{0}, t\right) \mathrm{d} \mu \leq \int_{S} \boldsymbol{e}\left(\omega_{0}, t\right) \mathrm{d} \mu$, and

(3.3) $u_{t}\left(\omega_{0}, \boldsymbol{y}\left(\omega_{0}, t\right)\right)>u_{t}\left(\omega_{0}, \boldsymbol{x}\left(\omega_{0}, t\right)\right)$ for almost all $t \in S$.

An allocation $\boldsymbol{x}$ is an ex-post core allocation if no coalition $S \in \Sigma$ has an ex-post improvement upon $\boldsymbol{x}$ at any $\omega \in \Omega$. The ex-post core of $\mathcal{E}$, denoted by $C(\mathcal{E})$, is the set of all the ex-post core allocations of $\mathcal{E}$. 
Theorem 3.1. If an economy $\mathcal{E}$ satisfies (A.1) and (A.3), then the ex-post core of $\mathcal{E}$ is non-empty. Moreover, the ex-post core of $\mathcal{E}$ is

$$
C(\mathcal{E})=\{\boldsymbol{x} \mid \boldsymbol{x} \text { is an assignment and } \boldsymbol{x}(\omega, \cdot) \in C(\mathcal{E}(\omega)) \text { for all } \omega \in \Omega\} .
$$

Proof. Let

$$
X=\{\boldsymbol{x} \mid \boldsymbol{x} \text { is an assignment and } \boldsymbol{x}(\omega, \cdot) \in C(\mathcal{E}(\omega)) \text { for all } \omega \in \Omega\} .
$$

We first show that $X \neq \emptyset$, and then we show that $C(\mathcal{E})=X$. It is well known that if (A.1) and (A.3) are satisfied, then $C(\mathcal{E}(\omega)) \neq \emptyset$, for all $\omega \in \Omega$ (see, e.g., Aumann, 1964, 1966, and Hildenbrand, 1968, 1974). Let $A_{1}, \ldots, A_{k}$ be all the atoms of the field $\mathcal{F}$. For every $1 \leq j \leq k$, let $\omega_{j} \in A_{j}$ and $\boldsymbol{x}_{j} \in C\left(\mathcal{E}\left(\omega_{j}\right)\right)$. Define $\boldsymbol{x}: \Omega \times T \rightarrow \mathcal{R}_{+}^{l}$ by $\boldsymbol{x}(\omega, t)=\boldsymbol{x}_{j}(t)$, whenever $\omega \in A_{j}$ and $t \in T$. Then $\boldsymbol{x}$ is a well-defined assignment in $\mathcal{E}$. Let $1 \leq j \leq k$ and $\omega \in A_{j}$. Then $\boldsymbol{e}(\omega, \cdot)=\boldsymbol{e}\left(\omega_{j}, \cdot\right)$, and for all $t \in T, u_{t}(\omega, \cdot)=u_{t}\left(\omega_{j}, \cdot\right)$. Therefore, $\mathcal{E}(\omega)=\mathcal{E}\left(\omega_{j}\right)$. Thus, $\boldsymbol{x}(\omega, \cdot)=\boldsymbol{x}\left(\omega_{j}, \cdot\right)$, which implies that $\boldsymbol{x}(\omega, \cdot) \in C(\mathcal{E}(\omega))$ for all $\omega \in \Omega$; hence, $\boldsymbol{x} \in X$ and $X \neq \emptyset$.

The proof that $C(\mathcal{E}) \supseteq X$ is straightforward. We show that $C(\mathcal{E}) \subseteq X$. Let $x \in C(\mathcal{E})$ and assume, contrary to our claim, that $\boldsymbol{x} \notin X$. Then there exists $\omega_{0} \in \Omega$ such that $\boldsymbol{x}\left(\omega_{0}, \cdot\right) \notin C\left(\mathcal{E}\left(\omega_{0}\right)\right)$. Therefore, there exists a coalition $S \in \Sigma$ with $\mu(S)>0$, and an integrable function $y: T \rightarrow \mathcal{R}_{+}^{l}$ such that $\int_{S} \boldsymbol{y}(t) \mathrm{d} \mu \leq \int_{S} \boldsymbol{e}\left(\omega_{0}, t\right) \mathrm{d} \mu$ and $u_{t}\left(\omega_{0}, \boldsymbol{y}(t)\right)>u_{t}\left(\omega_{0}, \boldsymbol{x}\left(\omega_{0}, t\right)\right)$ for almost all $t \in S$. Let $A\left(\omega_{0}\right)$ be the atom of the field $\mathcal{F}$ containing $\omega_{0}$. Define a function $z: \Omega \times T \rightarrow \mathcal{R}_{+}^{l}$ by

$$
z(\omega, t)= \begin{cases}\boldsymbol{y}(t) & \text { if } \omega \in A\left(\omega_{0}\right) \\ \boldsymbol{e}(\omega, t) & \text { otherwise }\end{cases}
$$

Then $\boldsymbol{z}$ is an assignment in $\mathcal{E}$. Moreover, $z$ is an ex-post improvement of $S$ upon $\boldsymbol{x}$ at $\omega_{0}$, which contradicts that $\boldsymbol{x} \in C(\mathcal{E})$.

\section{Rational expectations equilibria and the ex-post core}

In this section we study the relation between the ex-post core and the set of rational expectations equilibrium allocations.

Let $\mathcal{E}$ be an economy. If $\mathcal{G}$ is a subfield of $\mathcal{F}, f: \Omega \rightarrow \mathcal{R}_{+}$is an $\mathcal{F}$-measurable function, and $t \in T$, we denote by $E_{t}(f \mid \mathcal{G})$ the conditional expectation of $f$ with respect to $q_{t}$. A price system is an $\mathcal{F}$-measurable non-zero function $p: \Omega \rightarrow \mathcal{R}_{+}^{l}$. If $p$ is a price system, we denote by $\sigma(p)$ the smallest subfield $\mathcal{G}$ of $\mathcal{F}$ for which $p$ is $\mathcal{G}$-measurable. Note that the atoms of $\sigma(p)$ are the elements of the partition of $\Omega$ generated by the function $p$. The budget set of a trader $t \in T$ at the state of nature $\omega \in \Omega$ when the price system is $p$ is given by

$$
B_{t}(\omega, p)=\left\{a \in \mathfrak{R}_{+}^{l} \mid p(\omega) a \leq p(\omega) \boldsymbol{e}(\omega, t)\right\} .
$$

A rational expectations equilibrium is a pair $(p, \boldsymbol{x})$, where $p$ is a price system and $\boldsymbol{x}$ is an allocation such that

(4.1) for almost all $t \in T, \boldsymbol{x}(\cdot, t)$ is $\sigma(p) \vee \mathcal{F}_{t}$-measurable;

(4.2) for every $\omega \in \Omega$ and almost all $t \in T, \boldsymbol{x}(\omega, t) \in B_{t}(\omega, p)$; and 
(4.3) for almost all $t \in T$, if $\boldsymbol{y}: \Omega \rightarrow \mathcal{R}_{+}^{l}$ is $\sigma(p) \vee \mathcal{F}_{t}$-measurable and satisfies $\boldsymbol{y}(\omega) \in B_{t}(\omega$, p) for all $\omega \in \Omega$, then

$$
E_{t}\left(u_{t}(\cdot, \boldsymbol{x}(\cdot, t)) \mid \sigma(p) \vee \mathcal{F}_{t}\right) \geq E_{t}\left(u_{t}(\cdot, \boldsymbol{x}(\cdot)) \mid \sigma(p) \vee \mathcal{F}_{t}\right),
$$

pointwise on $\Omega$.

A rational expectations equilibrium $(p, \boldsymbol{x})$ is fully revealing if $\sigma(p)=\mathcal{F}$.

Proposition 4.1. Let $\mathcal{E}$ be an economy, and let $(p, \boldsymbol{x})$ be a fully revealing rational expectations equilibrium for $\mathcal{E}$. Then $\boldsymbol{x}$ is an ex-post core allocation of $\mathcal{E}$.

Proof. Let $\mathcal{E}$ be an economy, and let $(p, \boldsymbol{x})$ be a fully revealing rational expectations equilibrium for $\mathcal{E}$. Then $\sigma(p)=\mathcal{F}$. Since for every assignment $z$ we have

$$
E_{t}\left(u_{t}(\cdot, \boldsymbol{z}(\cdot, t)) \mid \sigma(p) \vee \mathcal{F}_{t}\right)=u_{t}(\cdot, z(\cdot, t)),
$$

for all $t \in T$, the proof that $x \in C(\mathcal{E})$ is completely analogous to the proof that every competitive allocation of a complete information economy is in the core.

The following example shows that there are rational expectations equilibrium allocations that are neither fully revealing nor ex-post core allocations.

Example 4.2. Consider an economy $\mathcal{E}$ in which the set of traders is $T=\{1,2\}$, the commodity space is $\mathcal{R}_{+}^{2}$, the space of states of nature is $\Omega=\left\{\omega_{1}, \omega_{2}\right\}$, and $\mathcal{F}=2^{\Omega}$. The traders have a common prior $q=(1 / 2,1 / 2)$. The information partition of Trader 1 is $\Pi_{1}=\left\{\left\{\omega_{1}, \omega_{2}\right\}\right\}$, and that of Trader 2 is $\Pi_{2}=\left\{\left\{\omega_{1}\right\},\left\{\omega_{2}\right\}\right\}$. Their initial endowments are $\boldsymbol{e}\left(\omega_{1}, 1\right)=\boldsymbol{e}\left(\omega_{2}, 1\right)=(2$, $4)$ and $\boldsymbol{e}\left(\omega_{1}, 2\right)=\boldsymbol{e}\left(\omega_{2}, 2\right)=(4,2)$, and their utility functions are $u_{1}\left(\omega_{1},(x, y)\right)=\sqrt{x}+2 \sqrt{y}$, $u_{1}\left(\omega_{2},(x, y)\right)=2 \sqrt{x}+\sqrt{y}$ and $u_{2}\left(\omega_{1},(x, y)\right)=u_{2}\left(\omega_{1},(x, y)\right)=\sqrt{x}+\sqrt{y}$, respectively. Let $p$ be the price system given by $p(\omega)=(1,1)$ for all $\omega \in \Omega$, and define the allocation $x$ by $x(\omega$, $t)=(3,3)$ for all $(\omega, t) \in \Omega \times T$. It is easy to check that $(p, x)$ is a rational expectations equilibrium for $\mathcal{E}$, and since $\sigma(p)=\left\{0,\left\{\omega_{1}, \omega_{2}\right\}\right\}$, it is not a fully revealing rational expectations equilibrium. The allocation $x$, however, is not an ex-post core allocation of $\mathcal{E}$. Indeed, the initial assignment $\boldsymbol{e}$ satisfies

$$
u_{1}\left(\omega_{1}, e\left(\omega_{1}, 1\right)\right)=\sqrt{2}+4>3 \sqrt{3}=u_{1}\left(\omega_{1}, x\left(\omega_{1}, 1\right)\right)
$$

so that Trader 1 blocks $\boldsymbol{x}$.

There are examples in the literature of economies satisfying the conditions of Theorem 3.1, but which do not have any rational expectations equilibrium — see, e.g., Kreps (1977), and Allen (1986). Therefore, in these economies, there are ex-post core allocations, which are not rational expectations equilibrium allocations.

Note that in Example 4.2 the utility function of Trader 1 is not measurable with respect to his information field. As we shall see, when each trader knows his state-dependent utility function (i.e., when each trader's utility function is measurable with respect to his information field), every rational expectations equilibrium allocation is an ex-post core allocation. 
Given an economy $\mathcal{E}$, we denote by $R E(\mathcal{E})$ the set of rational expectations equilibrium allocations of $\mathcal{E}$, and for $\omega \in \Omega$, we write $W(\mathcal{E}(\omega))$ for the set of competitive allocations of $\mathcal{E}(\omega)$.

Theorem 4.3. If an economy $\mathcal{E}$ satisfies (A.1)-(A.3), and (A.5), then

$R E(\mathcal{E})=\{\boldsymbol{x} \mid \boldsymbol{x}$ is an assignment and $\boldsymbol{x}(\omega, \cdot) \in W(\mathcal{E}(\omega))$ for all $\omega \in \Omega\}$.

\section{Proof. Let}

$Y=\{x \mid x$ is an assignment and $\boldsymbol{x}(\omega, \cdot) \in W(\mathcal{E}(\omega))$ for all $\omega \in \Omega\}$.

We first prove that $R E(\mathcal{E}) \subseteq Y$. Let $x \in \operatorname{RE}(\mathcal{E})$. We show that $x \in Y$. Let $\hat{\omega} \in \Omega$. We prove that $\boldsymbol{x}(\hat{\omega}, \cdot) \in W(\mathcal{E}(\hat{\omega}))$. Let $p$ be a price system such that $(p, \boldsymbol{x})$ is a rational expectations equilibrium of $\mathcal{E}$. We show that $(p(\hat{\omega}), \boldsymbol{x}(\hat{\omega}, \cdot))$ is a competitive equilibrium of $\mathcal{E}(\hat{\omega})$. Clearly, $\boldsymbol{x}(\hat{\omega}, t) \in B_{t}(\hat{\omega}, p)$ for almost all $t \in T$. We prove that $\boldsymbol{x}(\hat{\omega}, t)$ maximizes $u_{t}(\hat{\omega}, \cdot)$ on $B_{t}(\hat{\omega}, p)$ for almost all $t \in T$, which establishes that $x(\hat{\omega}, \cdot) \in W(\mathcal{E}(\hat{\omega}))$. Let $S$ be the set of all $t \in T$ such that (4.3) is satisfied for $(p, \boldsymbol{x})$. Then $\mu(S)=\mu(T)$. Let $t \in S$, and let $a \in B_{t}(\hat{\omega}, p)$. Denote by $A_{t}(\hat{\omega})$ the atom of $\sigma(p) \vee \mathcal{F}_{t}$ containing $\hat{\omega}$. Define $y: \Omega \rightarrow \mathcal{R}_{+}^{l}$ by

$$
y(\omega)= \begin{cases}a & \text { if } \omega \in A_{t}(\hat{\omega}) \\ 0 & \text { otherwise }\end{cases}
$$

Then $\boldsymbol{y}$ is a $\sigma(p) \vee \mathcal{F}_{t}$-measurable function. As $p(\omega)=p(\hat{\omega})$ and $\boldsymbol{e}(\omega, t)=e(\hat{\omega}, t)$ for all $\omega \in A_{t}(\hat{\omega})$, we have $\boldsymbol{y}(\omega) \in B_{t}(\omega, p)$ for all $\omega \in \Omega$. Since $t \in S$, we have by (4.3)

$$
E_{t}\left(u_{t}(\cdot, \boldsymbol{y}(\cdot)) \mid \sigma(p) \vee \mathcal{F}_{t}\right)(\hat{\omega}) \leq E_{t}\left(u_{t}(\cdot, \boldsymbol{x}(\cdot, t)) \mid \sigma(p) \vee \mathcal{F}_{t}\right)(\hat{\omega}) .
$$

By (A.3)

$$
E_{t}\left(u_{t}(\cdot, \boldsymbol{y}(\cdot)) \mid \sigma(p) \vee \mathcal{F}_{t}\right)=u_{t}(\cdot, \boldsymbol{y}(\cdot)),
$$

and

$$
E_{t}\left(u_{t}(\cdot, \boldsymbol{x}(\cdot, t)) \mid \sigma(p) \vee \mathcal{F}_{t}\right)=u_{t}(\cdot, \boldsymbol{x}(\cdot, t)) .
$$

Therefore

$$
u_{t}(\hat{\omega}, a)=u_{t}(\hat{\omega}, \boldsymbol{y}(\hat{\omega})) \leq u_{t}(\hat{\omega}, \boldsymbol{x}(\hat{\omega}, t)) .
$$

Thus, $\boldsymbol{x}(\hat{\omega}, t)$ maximizes $u_{t}(\hat{\omega}, \cdot)$ on $B_{t}(\hat{\omega}, p)$ for all $t \in S$. Since $\mu(S)=\mu(T)$, we have $\boldsymbol{x}(\hat{\omega}, \cdot) \in W(\mathcal{E}(\hat{\omega}))$.

It remains to be shown that $Y \subseteq R E(\mathcal{E})$. Let $\boldsymbol{x} \in Y$. Then $\boldsymbol{x}$ is an allocation in $\mathcal{E}$, and for all $\omega \in \Omega, \boldsymbol{x}(\omega, \cdot) \in W(\mathcal{E}(\omega))$. Therefore, for each $\omega \in \Omega$, there is a $p(\omega) \in \mathcal{R}_{+}^{l}$ such that $(p(\omega)$, $\boldsymbol{x}(\omega, \cdot))$ is a competitive equilibrium for $\mathcal{E}(\omega)$. Since for all $t \in T$ and $\omega \in \Omega$, the function $u_{t}(\omega, \cdot)$ is strictly increasing on $\mathcal{R}_{+}^{l}$ by (A.5), we have $p(\omega) \gg 0$, for all $\omega \in \Omega$. Let $A_{1}, \ldots, A_{k}$ be all the atoms of the field $\mathcal{F}$. For every $1 \leq j \leq k$, let $\omega_{j} \in A_{j}$. Define the function $\hat{p}: \Omega \rightarrow \mathcal{R}_{+}^{l}$ by $\hat{p}(\omega)=p\left(\omega_{j}\right)$, whenever $\omega \in A_{j}$. Then $\hat{p}$ is $\mathcal{F}$-measurable. We show that $(\hat{p}, \boldsymbol{x})$ is a rational expectations equilibrium for $\mathcal{E}$, and this will give $\boldsymbol{x} \in R E(\mathcal{E})$. We first 
claim that for all $\omega \in \Omega,(\hat{p}(\omega), \boldsymbol{x}(\omega, \cdot))$ is a competitive equilibrium for $\mathcal{E}(\omega)$. Let $\omega \in \Omega$; then there exists $1 \leq j \leq k$ and $\omega_{j} \in A_{j}$ such that $\hat{p}(\omega)=p\left(\omega_{j}\right)$. Now (A.2) and (A.3) imply that $\mathcal{E}(\omega)=\mathcal{E}\left(\omega_{j}\right)$. Also, since $\boldsymbol{x}$ is an assignment, we have $\boldsymbol{x}(\omega, \cdot)=\boldsymbol{x}\left(\omega_{j}, \cdot\right)$. As $\left(p\left(\omega_{j}\right), \boldsymbol{x}\left(\omega_{j}, \cdot\right)\right)$ is a competitive equilibrium for $\mathcal{E}\left(\omega_{j}\right)$, we have $(\hat{p}(\omega), \boldsymbol{x}(\omega, \cdot))$ is a competitive equilibrium for $\mathcal{E}(\omega)$.

We now prove that for all $t \in T, \boldsymbol{x}(\cdot, t)$ is $\sigma(\hat{p}) \vee \mathcal{F}_{t}$-measurable, i.e., that $\boldsymbol{x}(\cdot, t)$ is constant on the atoms of $\sigma(\hat{p}) \vee \mathcal{F}_{t}$. Let $t \in T$ and let $A$ be an atom of $\sigma(\hat{p}) \vee \mathcal{F}_{t}$. Assume that $\omega_{1}, \omega_{2} \in A$; then $\hat{p}\left(\omega_{1}\right)=\hat{p}\left(\omega_{2}\right)$. Since $e(\cdot, t)$ is $\mathcal{F}_{t}$-measurable, we have $\boldsymbol{e}\left(\omega_{1}, t\right)=\boldsymbol{e}\left(\omega_{2}, t\right)$. Therefore,

$$
B_{t}\left(\omega_{1}, \hat{p}\right)=B_{t}\left(\omega_{2}, \hat{p}\right) .
$$

Now by (A.3), we have

$$
u_{t}\left(\omega_{1}, \cdot\right)=u_{t}\left(\omega_{2}, \cdot\right) .
$$

Since $\left(\hat{p}\left(\omega_{1}\right), \boldsymbol{x}\left(\omega_{1}, \cdot\right)\right)$ and $\left(\hat{p}\left(\omega_{2}\right), \boldsymbol{x}\left(\omega_{2}, \cdot\right)\right)$ are competitive equilibria of $\mathcal{E}\left(\omega_{1}\right)$ and $\mathcal{E}\left(\omega_{2}\right)$, respectively, by (4.6) and (4.7), $\boldsymbol{x}\left(\omega_{1}, t\right)$ and $\boldsymbol{x}\left(\omega_{2}, t\right)$ are maximizers of $u_{t}\left(\omega_{1}, \cdot\right)$ on $B_{t}\left(\omega_{1}, \hat{p}\right)$. As $u_{t}\left(\omega_{1}, \cdot\right)$ is strictly quasi concave, it has a unique maximizer on $B_{t}\left(\omega_{1}, \hat{p}\right)$, and thus $\boldsymbol{x}\left(\omega_{1}, t\right)=\boldsymbol{x}\left(\omega_{2}, t\right)$, which establishes that $\boldsymbol{x}(\cdot, t)$ is $\sigma(\hat{p}) \vee \mathcal{F}_{t}$-measurable.

Since for all $\omega \in \Omega,(\hat{p}(\omega), \boldsymbol{x}(\omega, \cdot))$ is a competitive equilibrium of $\mathcal{E}(\omega)$, we have $\boldsymbol{x}(\omega, t) \in B_{t}(\omega, \hat{p})$ for all $\omega \in \Omega$ and almost all $t \in T$. Therefore ( $\left.\hat{p}, \boldsymbol{x}\right)$ satisfies (4.2).

It remains to be shown that $(\hat{p}, \boldsymbol{x})$ satisfies (4.3). For every $\omega \in \Omega$, let $S(\omega)$ be the set of all $t \in T$ such that $\boldsymbol{x}(\omega, t)$ maximizes $u_{t}(\omega, \cdot)$ on $B_{t}(\omega, \hat{p})$. Then $\mu(S(\omega))=\mu(T)$. Let $S=\cap_{\omega \in \Omega} S(\omega)$. Then $\mu(S)=\mu(T)$. Let $t \in S$ and let $y: \Omega \rightarrow \mathcal{R}_{+}^{l}$ be a $\sigma(\hat{p}) \vee \mathcal{F}_{t}$-measurable function such that $\boldsymbol{y}(\omega) \in B_{t}(\omega, \hat{p})$ for all $\omega \in \Omega$. Then, for all $\omega \in \Omega$, we have $u_{t}(\omega, \boldsymbol{y}(\omega)) \leq u_{t}(\omega, \boldsymbol{x}(\omega, t))$, and therefore

$$
E_{t}\left(u_{t}(\cdot, \boldsymbol{y}(\cdot)) \mid \sigma(\hat{p}) \vee \mathcal{F}_{t}\right) \leq E_{t}\left(u_{t}(\cdot, \boldsymbol{x}(\cdot, t)) \mid \sigma(\hat{p}) \vee \mathcal{F}_{t}\right)
$$

on $\Omega$. Since this inequality holds for all $t \in S,(\hat{p}, \boldsymbol{x})$ satisfies (4.3).

Corollary 4.4. If an economy $\mathcal{E}$ satisfies (A.1)-(A.3), and (A.5), then every rational expectations equilibrium allocation of $\mathcal{E}$ is in the ex-post core of $\mathcal{E}($ i.e., $R E(\mathcal{E}) \subseteq C(\mathcal{E})$ ).

Proof. Since $W(\mathcal{E}(\omega)) \subseteq C(\mathcal{E}(\omega))$ for all $\omega \in \Omega$, Corollary 4.4 follows from Theorems 3.1 and 4.3.

Theorem 4.5. Let $\mathcal{E}$ be an atomless economy (that is, such that the measure $\mu$ on $(T, \Sigma)$ is non-atomic) satisfying (A.1)-(A.3) and (A.5). Then, the set of rational expectations equilibrium allocations of $\mathcal{E}$ coincides with the ex-post core of $\mathcal{E}($ i.e., $R E(\mathcal{E})=C(\mathcal{E}))$.

Proof. By Aumann's Core-Equivalence Theorem, see Aumann (1964), for all $\omega \in \Omega$, $W(\mathcal{E}(\omega))=C(\mathcal{E}(\omega))$. Therefore, by Theorems 3.1 and 4.3, we have $\operatorname{RE}(\mathcal{E})=C(\mathcal{E})$.

Let $\mathcal{E}$ be a finite economy, i.e., $T=\{1, \ldots, n\}, \Sigma=2^{T}$, and $\mu$ is the counting measure. The $k$-fold replication of the economy $\mathcal{E}$ is an economy $\mathcal{E}^{k}$ in which the set of traders $T^{k}$ has $n k$ traders, the set of coalitions is the set of all subsets of $T^{k}$, and also there is a partition of $T^{k}$ to $n$ disjoint sets $T_{1}^{k}, \ldots, T_{n}^{k}$ such that for every $1 \leq i \leq n$, the traders in $T_{i}^{k}$ have the same utility, 
the same initial endowment, the same information field, and the same priors. An allocation $\hat{\boldsymbol{x}}$ in the economy $\mathcal{E}^{k}$ is an equal treatment allocation if it assigns the same random bundle to traders in the same set $T_{i}^{k}, 1 \leq i \leq n$; that is, if $t_{1}, t_{2} \in T_{i}^{k}$ for some $1 \leq i \leq n$, then

$$
\hat{\boldsymbol{x}}\left(\omega, t_{1}\right)=\hat{\boldsymbol{x}}\left(\omega, t_{2}\right)
$$

for all $\omega \in \Omega$. Let $k$ be a natural number and let $\hat{x}$ be an equal treatment allocation in $\mathcal{E}^{k}$. For $1 \leq i \leq n$, denote by $\hat{\boldsymbol{x}}(\cdot, i)$ the random bundle that $\hat{\boldsymbol{x}}$ assigns to the traders in $T_{i}^{k}$. Define the allocation $\boldsymbol{x}$ in $\mathcal{E}$ by $\boldsymbol{x}(\omega, t)=\hat{\boldsymbol{x}}(\omega, i)$ whenever $\omega \in \Omega$ and $t \in T_{i}$. We call $\boldsymbol{x}$ the allocation in $\mathcal{E}$ which corresponds to $\hat{\boldsymbol{x}}$. For every natural number $k$, we denote by $C\left(\mathcal{E}^{k}\right)$ the set of all allocations in $\mathcal{E}$ which corresponds to equal treatment ex-post core allocations in $\mathcal{E}^{k}$-such allocations exist by Theorem 3.1 and Debreu Scarf's Theorem; see Debreu and Scarf (1963).

Theorem 4.6. Let $\mathcal{E}$ be an economy with a finite number of traders satisfying (A.1)-(A.4). Then $\operatorname{RE}(\mathcal{E})=\bigcap_{k=1}^{\infty} C\left(\mathcal{E}^{k}\right)$.

Proof. Let $\omega \in \Omega$. For every natural number $k$ denote by $C\left(\mathcal{E}^{k}(\omega)\right)$ the set of all core allocations in $\mathcal{E}(\omega)$ which corresponds to equal treatment allocations in $\mathcal{E}^{k}(\omega)$. By the Debreu-Scarf's Theorem,

$$
W(\mathcal{E}(\omega))=\bigcap_{k=1}^{\infty} C\left(\mathcal{E}^{k}(\omega)\right) .
$$

Therefore, by Theorem 3.1, we obtain that

$$
\bigcap_{k=1}^{\infty} C\left(\mathcal{E}^{k}\right)=\{\boldsymbol{x} \mid \boldsymbol{x} \text { is an assignment and } \boldsymbol{x}(\omega, \cdot) \in W(\mathcal{E}(\omega)) \text { for all } \omega \in \Omega\} .
$$

Hence, by Theorem 4.3, $\operatorname{RE}(\mathcal{E})=\bigcap_{k=1}^{\infty} C\left(\mathcal{E}^{k}\right)$.

\section{Concluding remarks}

The ex-post core of an economy with asymmetric information does not depend on the information structure of the economy. In some cases, however, the logical relation between the ex-post core and the set of rational expectations equilibrium allocations of an economy, and the relation of these sets with other solution concepts studied in the literature, does depend on the information structure. Einy et al. (2000), for example, show that in an atomless economy with asymmetric information, Wilson's fine core is a subset of the ex-post core. Thus, Theorem 4.5, which we view as the main result in the present paper, implies that under assumptions (A.1)-(A.3) and (A.5), every allocation in Wilson's fine core is a rational expectations equilibrium allocation; see Corollary 3.4 in Einy et al. (2000).

Our results rely on the assumption that there is a finite number of states of nature. In fact, when there is an infinite number of states of nature some conceptual problems arise; it is not clear, for example, how to define the joint information of a coalition. 


\section{Acknowledgements}

We thank a co-editor and a referee for helpful comments. This work was done while Einy and Shitovitz visited the Department of Economics of the Universidad Carlos III de Madrid. Einy acknowledges the financial support of the Universidad Carlos III de Madrid. Moreno acknowledges the support of the Spanish Ministry of Education, grant PB97-0091. Shitovitz acknowledges the support of the Spanish Ministry of Education, grant SAB98-0059.

\section{References}

Allen, B., 1981. Generic existence of completely revealing equilibria for economies with uncertainty when prices convey information. Econometrica 49, 1173-1199.

Allen, B., 1982. Strict rational expectations equilibria with diffuseness. Journal of Economic Theory 27, 20-46.

Allen, B., 1986. General equilibirum with rational expectations. In: Hildenbrand, W., Mas-Collell, A. (Eds.), Contributions to Mathematical Economics in Honor of G. Debreu. North-Holland, Amsterdam.

Allen, B., 1997. Cooperative theory with incomplete information. In: Hart, S., Mas-Collell, A. (Eds.), Cooperation, Game Theoretic Approaches. Springer, Heidelberg.

Anderson, R.M., 1992. The core of perfectly competitive economies. In: Aumann, R.J., Hart, S. (Eds.), Handbook of Game Theory, vol. 1, North-Holland, Amsterdam.

Aumann, R.J., 1964. Markets with a continuum of traders. Econometrica 32, 39-50.

Aumann, R.J., 1966. Existence of competitive equilibria in markets with a continuum of traders. Econometrica $34,1-17$.

Debreu, G., Scarf, H., 1963. A limit theorem on the core of an economy. International Economic Review 4, 235-246.

Einy, E., Moreno, D., Shitovitz, B., 2000. On the Core of an Economy with Differential Information. Journal of Economic Theory.

Grossman, S.J., 1978. Further results on the informational efficiency of competitive stock markets. Journal of Economic Theory 18, 81-101.

Grossman, S.J., 1981. An introduction to the theory of rational expectations under asymmetric information. Review of Economic Studies 48, 541-559.

Hildenbrand, W., 1968. The core of an economy with a measure space of economic agents. Review of Economic Studies 35, 443-452.

Hildenbrand, W., 1974. Core and Equilibria of a Large Economy. Princeton Univ. Press, Princeton.

Kobayashi, 1980. Equilibrium contracts for syndicates with differential information. Econometrica 48, 1635-1665.

Koutsougeras, L.C., Yannelis, N.C., 1993. Incentive compatibility and information superiority of the core of an economy with differential information. Economic Theory 3, 195-216.

Kreps, D.M., 1977. A note on "fulfilled expectations" equilibria. Journal of Economic Theory 14, $32-43$.

Radner, R., 1979. Rational expectations equilibrium: generic existence and the information revealed by prices. Econometrica 47, 655-678.

Radner, R., 1982. Equilibrium under uncertainty. In: Arrow, K.J., Intriligator, M.D. (Eds.), Handbook of Mathematical Economics, vol. II, North-Holland, Amsterdam.

Wilson, R., 1978. Information, efficiency, and the core of an economy. Econometrica 40, 807-816.

Yannelis, N.C., 1991. The core of an economy with differential information. Economic Theory 1, 183-198. 